\title{
Impulsgeberin und Anstifterin: Die Deutsche Stiftung Friedensforschung im Lichte der Evaluation
}

\author{
Ulrich Schneckener • Thomas Held
}

Online publiziert: 24. April 2020

(C) Der/die Autor(en) 2020

Zusammenfassung Der Beitrag bewertet die Evaluation durch den Wissenschaftsrat aus Sicht der Deutschen Stiftung Friedensforschung (DSF). Er stellt die Rolle der DSF für das Forschungsfeld dar und diskutiert die wichtigsten Empfehlungen des Wissenschaftsrates für die Profilierung und die Weiterentwicklung der DSF. AuBerdem macht der Artikel deutlich, welche Perspektiven sich für die Förderung der Friedens- und Konfliktforschung ergeben - dies betrifft sowohl Förderstrukturen und Förderformate als auch die künftige finanzielle Ausstattung der DSF.

Schlüsselwörter Deutsche Stiftung Friedensforschung · Forschungsförderung · Finanzressourcen

U. Schneckener $(\bowtie)$

Zentrum für Demokratie- und Friedensforschung, Universität Osnabrück, Seminarstr.

33, 49069 Osnabrück, Deutschland

E-Mail: ulrich.schneckener@uni-osnabrueck.de

T. Held

Deutsche Stiftung Friedensforschung, Am Ledenhof 3-5, 49074 Osnabrück, Deutschland

E-Mail: theld@bundesstiftung-friedensforschung.de 


\title{
Initiating and stimulating research. The German Foundation for Peace Research in the light of the evaluation
}

\begin{abstract}
The article examines the evaluation by the German Science Council from the perspective of the German Foundation for Peace Research (DSF). It analyzes the role of the foundation for the research area as well as the most important recommendations of the Science Council for the future development of the DSF. The contribution explores furthermore the funding perspectives for peace and conflict research which includes funding structures and formats as well as the future financial resources of the DSF.
\end{abstract}

Keywords German Foundation for Peace Research · Research funding · Financial resources

\section{Genese und Ziele der Evaluation}

Die Evaluation des Wissenschaftsrates hatte für die Deutsche Stiftung Friedensforschung (DSF) als Einrichtung der Forschungsförderung eine besondere Bedeutung: Die DSF war die einzige Institution des Forschungsfeldes, die gezielt mit Blick auf ihre Stärken und Schwächen sowie ihre Zukunftsperspektiven bewertet wurde, was sich in einem eigenen Berichtsteil in der Gesamtevaluation niederschlug. Diese Ausgangslage ergab sich bereits aus dem Beschluss des Deutschen Bundestages vom Januar 2017 „Dem Frieden verpflichtet - Friedens- und Konfliktforschung stärken “ (Deutscher Bundestag 2017), der einer - nicht ganz einfachen - Kompromissfindung innerhalb der damaligen Regierungsfraktionen von CDU/CSU und SPD folgte. In dem Antrag wurde zwar der Beitrag der Forschung zur Friedenssicherung, Gewaltund Krisenprävention sowie zur Konfliktlösung für Regierung, Parlament und Gesellschaft als „unverzichtbar“ gewürdigt. Daraus folgten jedoch keine konkreten Maßnahmen oder Fördermittel, um die Friedens- und Konfliktforschung strukturell zu stärken. Stattdessen konnten sich die Koalitionsfraktionen lediglich darauf verständigen, das Forschungsfeld im Allgemeinen und die DSF im Besonderen durch den Wissenschaftsrat evaluieren zu lassen.

Auf Anfrage des Bundesministeriums für Bildung und Forschung (BMBF) nahm der Wissenschaftsrat die so genannte Strukturevaluation in sein Arbeitsprogramm auf, die in Empfehlungen und Optionen für die Weiterentwicklung des Feldes münden sollte. Sie zielte nicht auf die Bewertung von Einzelinstitutionen - eben mit Ausnahme der DSF, die als feldspezifische Fördereinrichtung national wie international ein Alleinstellungsmerkmal besitzt. Ende 2017 nahm die eingesetzte Arbeitsgruppe der Gutachter*innen ihre Arbeit auf und organisierte 2018 diverse Treffen und Anhörungen mit verschiedenen Akteuren des Forschungsfeldes sowie mit the- 
matisch angrenzenden Institutionen. In einer Sitzung im Dezember 2018 wurde auch die DSF auf „Herz und Nieren“ geprüft. ${ }^{1}$

Der im Juli 2019 veröffentlichte Abschlussbericht (Wissenschaftsrat 2019) richtet sich - neben dem Forschungsfeld und der DSF - an die Auftraggeber im Bund, den Deutschen Bundestag, das BMBF und weitere relevante Fachressorts sowie an die für den Hochschulbereich zuständigen Länder. Alle Adressaten werden in unterschiedlicher Weise dazu aufgefordert, die Weiterentwicklung des Forschungsfeldes mit gezielten strukturbildenden Maßnahmen zu unterstützen. Um das Ergebnis vorwegzunehmen: Für die DSF fiel die Evaluation in doppelter Hinsicht sehr positiv aus. Der Wissenschaftsrat würdigte zum einen die strukturbildende Wirkung, die die DSF für das Forschungsfeld entfaltet hat, und zum anderen das Kerngeschäft der Forschungsförderung gemessen am Satzungsauftrag und an den vergleichsweise bescheidenen Ressourcen.

Die Bewertungen und Empfehlungen des Wissenschaftsrats haben ihre Wirkung im politischen Raum nicht verfehlt. In der Bundestagsdebatte am 13.02.2020 zu einem Beschlussantrag der Fraktion Bündnis90/Die Grünen (2019) begrüßten die Redner*innen aller Fraktionen (mit Ausnahme der AfD) das positive Ergebnis der Evaluation und bekannten sich zur Stärkung des Forschungsfeldes sowie der DSF. ${ }^{2}$ Der Parlamentarische Staatssekretär im BMBF, Dr. Michael Meister (CDU), stellte mit Blick auf die Friedens- und Konfliktforschung fest: „Es ist ein Glücksfall für den Standort Deutschland, dass wir sie haben“ (Deutscher Bundestag 2020). Zugleich kündigte er erste konkrete Fördermaßnahmen durch die Bundesregierung an.

Dieser Beitrag bewertet die Evaluation primär aus der Perspektive der DSF. Bevor er sich jedoch mit den stärker DSF-bezogenen Empfehlungen auseinandersetzt, ist es sinnvoll, die Stiftung und ihre Handlungsmöglichkeiten in die Strukturen des Forschungsfeldes einzuordnen und damit die Rahmenbedingungen ihrer Aktivitäten in Erinnerung zu rufen.

\section{Die Rolle der DSF im Forschungsfeld}

Die DSF wurde im Jahr 2000 durch den Bund gegründet, um vor dem Hintergrund historischer Erfahrungen die politische Unabhängigkeit einer feldspezifischen Forschungsförderung für die Friedens- und Konfliktforschung auf Dauer sicherzustellen. Aus diesem Grund entschied sich die damalige rot-grüne Bundesregierung für das Modell einer kapitalgedeckten Stiftung bürgerlichen Rechts, d.h. die Stiftung handelt unabhängig von laufenden Zuwendungen aus öffentlichen Haushalten und verwendet für die Finanzierung ihrer Förderprogramme und institutionellen Kosten nur die Erträge aus der Bewirtschaftung des Stiftungsvermögens. Dabei wurde der

\footnotetext{
1 Die Autoren dieses Beitrags vertraten die DSF für den Vorstand bzw. die Geschäftsstelle. Die stellvertretende Vorsitzende des Stiftungsrates, Prof. Dr. Cilja Harders (FU Berlin) sowie die damalige Vorsitzende des Wissenschaftlichen Beirates, Prof. Dr. Heike Krieger, nahmen aus Sicht der jeweiligen Gremien zur Arbeit der DSF Stellung.

2 Siehe hierzu Zusammenfassung der Debatte auf der Homepage der DSF (https://bundesstiftungfriedensforschung.de/blog/ein-kompass-in-unruhigen-zeiten-der-deutsche-bundestag-debattiert/ (Zugriff vom 13.4.2020))
} 
DSF grundsätzlich die Möglichkeit des Kapitalverzehrs - unter gewissen Auflagen - eingeräumt.

Der Kernauftrag der DSF besteht in der Förderung und Initiierung wissenschaftlicher Projekte, die von Forschungsvorhaben über Tagungen bis hin zu Vernetzungen reichen. Die Stiftung führt zwar selbst keine Untersuchungen durch, sie wirkt aber mit eigenen wissenschaftlichen Impulsen wie Förderinitiativen und Tagungen in das Forschungsfeld hinein. Die finanzielle Ausstattung der DSF in Höhe von 25,56 Mio. $€^{3}$ erwies sich schon kurz nach ihrer Gründung als deutlich zu niedrig, um eine dem Stiftungszweck angemessene Forschungsförderung aufzubauen. Bereits das von Anfang an geplante Sonderprogramm zur Struktur- und Nachwuchsförderung - die Anschubfinanzierung für Stiftungsprofessuren, Masterstudiengänge sowie eine strukturierte Doktorandenförderung in Höhe von rund 5 Mio. $€$ (2002 bis 2006) - musste vollständig aus dem Grundstockkapital finanziert werden. Diese Entnahme konnte später durch schrittweise Zustiftungen des Bundes wieder ausgeglichen werden, die strukturelle Unterkapitalisierung ist bis heute die Achillesferse der DSF.

In den Folgejahren suchte die Stiftung aus dieser Not eine Tugend zu machen, indem sie ihre strategische Positionierung im Forschungsfeld in drei Schritten neu ausrichtete: Erstens wurde 2013 das neue Förderkonzept „Forschungsinnovation, Netzwerkbildung, Praxistransfer" verabschiedet, das mit einer thematisch offenen Förderung auf besondere Bedarfe des Forschungsfeldes abzielte und die Alleinstellungsmerkmale in der Förderpraxis der DSF in den Vordergrund rückte. Dafür stellt die Stiftung heute eine jährliche Mindestfördersumme von $730.000 €$ bereit unabhängig von der aktuellen Ertragslage. ${ }^{4}$

Zweitens folgte 2016 eine umfassende Satzungsreform, mit der ein deutlich flexibleres Organisationsmodell umgesetzt wurde: Der neu besetzte fünfköpfige Vorstand führt eigenständig das operative Geschäft der Stiftung und trifft infolgedessen (fast) alle Förderentscheidungen. Der Stiftungsrat (12 Mitglieder aus Bundesministerien, dem Deutschen Bundestag und der Wissenschaft) konzentriert sich dagegen weitgehend auf Aufsichtsfunktionen und strategische Fragen. Zudem wurde der Stiftungszweck auch offiziell um den Bereich des Wissenstransfers erweitert.

Drittens ergänzte die DSF - eine der ersten Initiativen des Vorstands - die offene Förderung um eine thematische Förderlinie, die 2017 erstmalig zum Thema „Neue Technologien. Risiken und Chancen für internationale Sicherheit und Frieden " ausgeschrieben wurde. Verteilt über zwei Jahre standen für diesen Zweck zusätzliche Fördermittel von $600.000 €$ zur Verfügung, die die DSF aufgrund der Ertragslage nur über den Verzehr aus dem Stiftungskapital finanzieren konnte. ${ }^{5}$ Der Wissenschaftsrat bewertete das neue Förderinstrument sehr positiv. Aus diesem Grund stimmte der Stiftungsrat bereits im November 2019 zu, die thematische Förderlinie

\footnotetext{
3 Die ursprüngliche Planung im politischen Raum hatte ein Stiftungskapital von 50 Mio. $€$ vorgesehen.

4 Das Budget wurde 2019 durch Beschluss des Stiftungsrates von 640.000 auf $730.000 €$ angehoben, insbesondere um den gestiegenen Personalkosten Rechnung zu tragen.

5 Diese Entscheidung gründet auf einer Regelung in der Stiftungssatzung in § 4(2), die es der DSF erlaubt, für besondere Förderzwecke einen Anteil des Stiftungskapitals in Höhe von bis zu 2,5 Mio. € zu verzehren.
} 
als ein strategisches Profilelement der Forschungsförderung der DSF zu verstetigen. Derzeit ist eine erneute Ausschreibung der Förderlinie in Vorbereitung.

In der Summe beläuft sich das jährliche Fördervolumen der DSF gegenwärtig auf 1,04 Mio. $€$, das Gesamtbudget auf rund 1,5 Mio. $€$. Aufwendungen in dieser Höhe kann die Stiftung jedoch bei weitem nicht durch ihre Erträge aus dem Stiftungsvermögen decken. Die Hauptursache liegt in der niedrigen Kapitalausstattung der Stiftung; die anhaltende Niedrigzinsphase hat diese Situation in den vergangenen Jahren weiter verschärft. Die DSF sieht sich daher seit 2018 gezwungen, zur Aufrechterhaltung ihrer Förderleistungen das Stiftungskapital in stärkerem Maße anzuzapfen. Sollte diese Defizitfinanzierung in den kommenden Jahren anhalten, dürfte dies das erreichte Förderniveau und letztlich den Fortbestand der DSF in Frage stellen. Der Vorstand hat gegenüber dem Wissenschaftsrat auf die prekäre Lage hingewiesen, da die DSF absehbar vor der Alternative steht, entweder eine deutlich verbesserte Kapitalausstattung zu erhalten oder aber mittelfristig die geordnete Auflösung der Stiftung in die Wege leiten zu müssen. Die dritte Variante bestünde darin, das bestehende Vermögen nominal zu erhalten und nicht anzutasten - mit der Folge, dass kaum noch signifikante Förderaktivitäten entfaltet werden könnten, was den Stiftungszweck schlicht ad absurdum führen würde. Diese Option des faktischen Stillstands ist aber weder in die Wissenschaft noch in die Politik vermittelbar. Über letzteres besteht in den Gremien der DSF ein klarer Konsens.

\section{Die DSF in der Bewertung durch den Wissenschaftsrat}

Vor diesem Hintergrund musste sich der Wissenschaftsrat mit der Kernfrage befassen, ob und in welcher Form eine Fördereinrichtung wie die DSF in der Lage ist, die von ihr erwarteten Beiträge zur strategischen Fortentwicklung des Forschungsfeldes zu leisten. Die Evaluation hat diese Frage in zweifacher Hinsicht klar bejaht ${ }^{6}$ : Die DSF habe zum einen mit ihren Förderleistungen ,einen wesentlichen Beitrag zur Konsolidierung, Stärkung sowie konzeptionellen Weiterentwicklung“ (Wissenschaftsrat 2019, S. 9) der Friedens- und Konfliktforschung geleistet, nicht zuletzt durch die oben genannte Strukturförderung in den 2000er Jahren. ${ }^{7}$ Zum anderen habe sie ihre Fähigkeit zur Selbstreflexion unter Beweis gestellt, um Förderformate immer wieder neu auszurichten und damit kontinuierlich Impulse für Forschung, Vernetzung und Wissenstransfer zu setzen. Aufgrund ihrer spezifischen Kompetenzen und „einzigartigen Kenntnis des Forschungsfeldes“ (Wissenschaftsrat 2019, S. 157) sei die DSF für die Fortentwicklung der Friedens- und Konfliktforschung in Deutschland deshalb auch in der Zukunft unverzichtbar.

Der Wissenschaftsrat empfiehlt der DSF, den bereits eingeschlagenen Weg fortzusetzen, indem sie sich primär als Impulsgeberin und Anstifterin für innovative Pilotvorhaben und Projekte, interdisziplinäre und internationale Vernetzung sowie den Dialog mit Politik und Gesellschaft positioniert. In dieser Funktion sieht er die große

\footnotetext{
${ }^{6}$ Die Bewertung und die Empfehlungen zur DSF finden sich im Bericht zusammenfassend (Wissenschaftsrat 2019, S. 64-67) sowie im ausführlichen im Anhang 3 (Wissenschaftsrat 2019, S. 125-167).

7 Siehe hierzu auch den Beitrag von Bonacker (https://doi.org/10.1007/s42597-020-00028-z).
} 
Stärke der DSF, da sie ihre Angebote zielgerichtet und ergänzend zu anderen Fördermöglichkeiten entwickeln könne. Die Kombination aus offener und thematischer Förderung wurde ausdrücklich befürwortet, da sie der Stiftung über unterschiedliche Förderformate - Forschungsprojekte, Tagungen, Vernetzung und Transfer - hinweg einen flexiblen und an verschiedenen Bedarfen ausgerichteten Handlungsrahmen biete. Die Stiftung solle sich dabei noch intensiver darauf konzentrieren, die Förderangebote dafür einzusetzen, die Strategiefähigkeit des Forschungsfeldes insgesamt zu stärken.

Für ausbaufähig hält der Wissenschaftsrat die Scharnierfunktion der DSF für eine Stärkung der multi- und interdisziplinären Zusammenarbeit, die Einbindung von unterrepräsentierten Fachdisziplinen bzw. die Adressierung von Schnittstellen zu angrenzenden Forschungsfeldern, wie dies bspw. schon bei der Migrations- und Fluchtforschung oder Terrorismus- und Radikalisierungsforschung geschieht. Die Förderpraxis der DSF relativiert bis zu einem gewissen Grad den Befund des Wissenschaftsrates, wonach im Zuge der Professionalisierung des Forschungsfeldes eine disziplinäre Verengung auf die Politikwissenschaft, insbesondere auf das Teilgebiet der Internationalen Beziehungen, stattgefunden habe. Dies gilt zwar für tragende Institutionen des Feldes, bildet aber nicht die Breite der Forschungs- und Tagungsaktivitäten $\mathrm{ab}$, die über Jahre von der DSF in anderen Disziplinen wie z.B. dem Völkerrecht, der Pädagogik, der Sozialpsychologie, der Kommunikationswissenschaft, der Sozialanthropologie, den Naturwissenschaften sowie der Soziologie, der Philosophie und der Geschichtswissenschaft gefördert wurden. Eine wesentliche Herausforderung besteht gleichwohl darin, diese Forschungen innerhalb des Forschungsfeldes besser zu vernetzen und sichtbarer zu machen, um die ,unerlässliche Interdisziplinarität" zu sichern (Wissenschaftsrat 2019, S. 161). ${ }^{8}$ Mit der thematischen Offenheit ihres Förderkonzepts versuche die DSF, wie der Wissenschaftsrat explizit betonte, dazu einen Beitrag zu leisten. Außerdem könne die DSF mit ihren Formaten - möglicherweise stärker als andere - explorative und synthetisierende Projekte unterstützen.

Die Verstetigung der thematischen Förderlinie wird als ein wichtiges profilbildendes Element für die DSF betrachtet, mit dem sowohl auf politische und gesellschaftliche Herausforderungen als auch auf neue Forschungsfelder reagiert werden kann. Trotz des positiven Gesamturteils zur Fördertätigkeit sieht der Wissenschaftsrat noch Potenziale für eine stärkere Kohärenz und Fokussierung der DSF-Formate. Die Aufforderung lautet hier: Noch mehr auf die Initiierung von innovativen Pilotvorhaben zu setzen sowie die Tagungs- und Vernetzungsförderung aktiver für strategische Ziele wie interdisziplinäre und internationale Zusammenarbeit zu nutzen.

Bei der Nachwuchsförderung empfiehlt der Wissenschaftsrat hingegen aufgrund von bestehenden Alternativen, das 2013 eingeführte Post-Doc Projekt wieder aus dem Sortiment zu nehmen. Bei diesem Format hatte die Stiftung in der Tat Anlaufschwierigkeiten, da es zwar eine ausreichende Zahl an Anträgen, aber nach der Begutachtung kaum förderungswürdige Projekte gab - erst 2018 konnten drei Post-

\footnotetext{
8 Siehe hierzu auch die Beiträge von Bonacker (https://doi.org/10.1007/s42597-020-00028-z) und Schröder/Niemann (https://doi.org/10.1007/s42597-020-00033-1) sowie aus der Perspektive der politischen Praxis von Gehring/Rubner (https://doi.org/10.1007/s42597-020-00031-3).
} 
Doc Projekte in die Förderung aufgenommen werden. Die DSF wird daher die Nachwuchsförderung - immerhin auch ein Satzungsauftrag - noch stärker als bisher zur Querschnittsaufgabe für alle Förderformate machen. Auch wenn es von Antragsteller*innen durchaus immer wieder Kritik am aufwändigen und zeitintensiven Begutachtungsverfahren gibt, begrüßt der Wissenschaftsrat ausdrücklich die bestehenden Prozeduren als ,,state of the art“ und „,qualitativ hochwertig“ (Wissenschaftsrat 2019, S. 66). Die DSF wird die einzelnen Empfehlungen zur Förderpraxis bei der anstehenden Überarbeitung und Weiterentwicklung des Förderkonzeptes (einschließlich des strategischen Leitbildes) berücksichtigen. Das neue Förderkonzept soll möglichst ab 2021 gelten und in der Fachcommunity publik gemacht werden.

Obgleich der Wissenschaftsrat die Strukturförderung aus der Anfangsphase der DSF sehr gelobt hat, finden sich im Bericht keine Empfehlungen zu diesem Punkt oder auch zu anderen möglichen Aufgabenfeldern, die bei einer entsprechenden Ressourcenausstattung von der DSF künftig übernommen werden könnten. Der Vorstand hat dazu schriftlich wie mündlich strategisch ausgerichtete Vorschläge gemacht (u.a. Anschubfinanzierung für Junior- und Stiftungsprofessoren, Maßnahmen zur Internationalisierung und zur Verbundforschung sowie zum Ausbau der Forschungsinfrastruktur), diese fanden jedoch keinen Niederschlag in den Empfehlungen für die DSF. Gleichwohl wird es eine Aufgabe und Herausforderung für die Stiftung bleiben, sich auch mit den für das Forschungsfeld relevanten Strukturproblemen auseinanderzusetzen und nach kreativen Lösungen sowie Kooperationspartnern bei der Finanzierung zu suchen.

Der Wissenschaftsrat beantwortete abschließend die Grundsatzfrage positiv, ob sich das Stiftungsmodell zur Forschungsförderung bewährt habe. Die Rechtsform als Stiftung bürgerlichen Rechts sichere die politische Unabhängigkeit der Fördertätigkeit, insbesondere in einem Forschungsfeld, das schon allein durch seinen Forschungsgegenstand in einer permanenten Wechselbeziehung mit dem politischen Raum stehe. Bei der künftigen Weiterentwicklung der DSF müsse daher der unabhängige Status der DSF unbedingt bewahrt werden.

Auf dieser Basis kommt der Wissenschaftsrat zu dem Schluss, dass die DSF für die Erfüllung ihres Stiftungszwecks finanziell dringend besser ausgestattet werden müsse. Dazu sollten die Bundesregierung und der Haushaltsgesetzgeber einen Weg wählen, der die Arbeitsfähigkeit der Stiftung auf lange Sicht auch für künftige Generationen von Wissenschaftler*innen sichert. Als präferierte Lösung empfiehlt der Wissenschaftsrat deshalb eine angemessene Aufstockung des Stiftungskapitals. Dabei vermeidet er es, einen konkreten Betrag für die Kapitalaufstockung zu benennen und schlägt stattdessen vor, als „Untergrenze“ ein jährliches Mindestförderbudget in Höhe von einer Million Euro anzusetzen (Wissenschaftsrat 2019, S. 66). Zusammen mit den Aufwendungen für den Wissenstransfer, für weitere operative Aktivitäten und für die Verwaltung der DSF wäre somit ein jährliches Gesamtbudget von ca. 1,5 Mio. $€ \mathrm{zu}$ finanzieren. Um eine solche „Untergrenze“ - anders als momentan - künftig und dauerhaft aus Erträgen finanzieren zu können, bedarf es einer deutlichen Erhöhung des Stiftungskapitals. Das jährliche Fördervolumen bliebe dabei beim derzeitigen Status quo, wäre aber nachhaltig finanziell abgesichert.

Die unterschiedlichen Lösungswege, um eine verbesserte finanzielle Ausstattung zu erreichen, sind an einem zentralen Grundsatz auszurichten: Sie müssen 
mit dem Stiftungsmodell konform sein, d.h. die DSF muss einen flexiblen, von politischen Konjunkturen und Abhängigkeiten sowie von kameralistischen Zwängen freien Handlungsrahmen für ihre Förderaktivitäten erhalten, um auch gegenüber anderen privaten oder staatlichen Fördereinrichtungen der Wissenschaft konkurrenzfähig zu bleiben. Der ideale Weg sind daher Zustiftungen aus dem Bundeshaushalt zum Kapitalstock, sei es, um kurzfristig das Defizit zu decken und den steigenden Kapitalverzehr zu stoppen, oder sei es, um mittelfristig das Vermögen schrittweise in eine Größenordnung aufzustocken, die den Anforderungen der Empfehlung des Wissenschaftsrates gerecht wird. Das BMBF als Vertreter der Stifterin im Stiftungsrat wird gebeten, ,zeitnah - spätestens in drei Jahren - über die Umsetzung der Empfehlungen zur DSF zu berichten“ (Wissenschaftsrat 2019, S. 67).

\section{Perspektiven für die DSF und das Forschungsfeld}

Was würde ein solcher Ausbau der DSF für die künftige Entwicklung der Forschungsförderung bzw. für die strukturelle Unterstützung des Forschungsfeldes bedeuten? Um gleich ein wenig Wasser in den Wein zu kippen: Selbst wenn man ein optimistisches Szenario zugrunde legt, wird die DSF auch künftig keine Fördereinrichtung sein, die wie die Deutsche Forschungsgemeinschaft (DFG) oder andere Förderer höhere Fördersummen für einzelne Projekte anbieten kann, mit denen sich längere Laufzeiten oder eine höhere Zahl an Personalstellen finanzieren lassen. Im günstigen Fall dürfte es möglich sein, die Projektbudgets etwas großzügiger zu bemessen. Die gezielte Förderung von größeren Verbundprojekten oder interdisziplinären Arbeitsgruppen wird auch unter verbesserten Bedingungen eine Herausforderung für die DSF bleiben. Dennoch eröffnet eine höhere Kapitalausstattung zweifelsohne bei einer entsprechenden Ertragslage und bei Beibehaltung der Option des (zeitweisen) Kapitalverzehrs auch Handlungsspielräume, die punktuell für solche Maßnahmen genutzt werden können.

Eine andere Frage ist allerdings, ob und inwieweit die DSF beratende oder koordinierende Aufgaben übernehmen kann, die die Empfehlungen des Wissenschaftsrates für das Forschungsfeld betreffen. Dies gilt insbesondere für den Aufbau von überregionalen, möglichst interdisziplinär angelegten, Verbundprojekten, was sich durch „kurzatmige“ Projektförderungen nicht realisieren lässt. Der Wissenschaftsrat sieht hier Bund und Länder in der Pflicht, entsprechende Förderprogramme mit längeren Zeiträumen und höheren Volumina aufzulegen. Grundsätzlich könnte die DSF für solche übergreifenden Förderprogramme ihre Förderinfrastruktur bereitstellen und wie sie es bei der Struktur- und Nachwuchsförderung in der Vergangenheit erfolgreich praktiziert hat - in Kooperation mit den universitären und außeruniversitären Einrichtungen geeignete Förderformate entwickeln.

Den größten unmittelbaren Handlungsbedarf sieht der Wissenschaftsrat im Bereich der natur- und technikwissenschaftlichen Friedens- und Konfliktforschung, die 
sich personell und institutionell in einer prekären Lage befindet. ${ }^{9}$ Es bestehe eine gravierende Diskrepanz zwischen dem Bedarf an Forschung und Politikberatung einerseits und den Forschungskapazitäten andererseits. Die Ausschreibung der DSFFörderlinie „Neue Technologien“ sei zwar anerkennungswert, sie könne jedoch das strukturelle Problem nicht lösen. Die vergleichsweise konkrete Empfehlung des Wissenschaftsrates, nämlich die Einrichtung von je zwei dauerhaften Leitungsstellen, ergänzt um befristete Qualifikationsstellen an mindestens zwei Standorten, dürften eine langfristige Absicherung der natur- und technikwissenschaftlichen Friedensforschung ermöglichen. Für die Umsetzung dieser Empfehlung werden nur wenige Standorte in Frage kommen. Dafür müssen entsprechende Konzepte vorgelegt werden, wie die naturwissenschaftlich-technische Forschung künftig besser mit der sozialwissenschaftlichen und völkerrechtlichen Forschung in den Feldern von Abrüstung, Rüstungskontrolle oder Proliferation - unter Berücksichtigung neuer Waffensysteme und jüngerer technologischer Entwicklungen - verzahnt werden kann. Die DSF kann hier bei Bedarf eine beratende und vermittelnde Funktion übernehmen, zumal die Stiftung seit mehreren Jahren auf die prekäre Situation in diesem Bereich hingewiesen hat.

Die unzureichende Grundfinanzierung der Forschungsinstitute wird vom Wissenschaftsrat eher allgemein thematisiert, konkreter wird nur eine stärkere Beteiligung des Bundes an den länderfinanzierten Einrichtungen von BICC und IFSH gefordert. Das Problem ist jedoch grundsätzlicher Natur und kann - trotz beachtlicher Erfolge - nicht auf Dauer durch hohe Drittmittelaufkommen kompensiert werden. Dies betrifft nicht nur den Erhalt und Ausbau von Forschungskapazitäten, sondern auch den vom Wissenschaftsrat als ,sehr engagiert“ und „kritisch reflektiert“ begrüßten Wissenstransfer in Gesellschaft und Politik (Wissenschaftsrat 2019, S. 9). ${ }^{10}$ Letzterer bedarf einer soliden Grundfinanzierung und professionalisierter Bereiche an den Forschungseinrichtungen. Die DSF plant bei der Überarbeitung ihres Förderformates zwar hier für Projektnehmer*innen zusätzliche Ressourcen zur Verfügung zu stellen bzw. eigene Transfertagungen zu organisieren, was aber keineswegs das Problem löst, solche Daueraufgaben zu finanzieren.

Darüber hinaus gibt es weitere Desiderate, die von der Evaluation angesprochen, aber nicht explizit mit Empfehlungen zu Ressourcen unterlegt werden. Aus Sicht der DSF sind hier drei Punkte für die künftige Entwicklung des Forschungsfeldes besonders relevant: Synthesefähigkeit, interdisziplinäre Anschlussfähigkeit sowie Internationalisierung.

Erstens muss dem Forschungsfeld daran gelegen sein, angesichts fortschreitender Spezialisierung und Binnendifferenzierung, Forschungsergebnisse zu bündeln und so etwas wie „Synthesewissen“ bereitzustellen. Eine solche Aufgabe läuft gewissermaßen quer zur Logik der etablierten Forschungsförderung, die auf die Identifikation neuer Forschungsfelder und die Schließung von Forschungslücken setzt, die es aber kaum erlaubt, Erträge jahrzehntelanger Forschung kritisch aufzuarbeiten und

\footnotetext{
${ }^{9}$ Siehe hierzu auch die Beiträge von Reuter/Altmann/Göttsche/Himmel (https://doi.org/10.1007/s42597020-00035-z), Schröder/Niemann (https://doi.org/10.1007/s42597-020-00033-1) und Gehring/Rubner (https://doi.org/10.1007/s42597-020-00031-3).

10 Zur Rolle des Wissenstransfers siehe auch den Beitrag von Schröder/Niemann (https://doi.org/10.1007/ s42597-020-00033-1).
} 
zusammenzuführen. Das Ergebnis ist eine zunehmend parzellierte, hochgradig spezialisierte Forschungslandschaft, bei der schon Insider Mühe haben, den Überblick zu behalten. Die Synthese-Funktion ist daher inner-wissenschaftlich von Bedeutung, um Wissensbestände zu sichern, in der Lehre zu vermitteln und darauf aufbauend innovative Fragestellungen zu entwickeln. Mehr noch: Für den Wissenstransfer und die Kommunikation mit der Öffentlichkeit ist diese Fähigkeit geradezu elementar, um Außenstehenden zumindest eine erste Orientierung vermitteln zu können. Die thematischen, interdisziplinär ausgerichteten Förderlinien der DSF können hier - je nach Zuschnitt - einen bescheidenen Beitrag leisten, gerade in der Zusammenschau der geförderten Projekte. Gleichwohl wird auch die Stiftung bei ihren Förderformaten künftig darüber nachdenken, wie Themenfelder fächerübergreifend ausgewertet und für Forschung und Praxis aufgearbeitet werden können.

Zweitens gilt es die disziplinäre Breite des Forschungsfeldes zu erhalten und auszubauen. In manchen Fächern wie der Wirtschaftswissenschaft ist die Friedens- und Konfliktforschung so gut wie gar nicht vertreten. Auch in anderen Fachgebieten wie bspw. der Psychologie oder den Erziehungswissenschaften - Stichwort Friedenspädagogik - fehlen grundständige Professuren mit Schwerpunkten in der Friedensund Konfliktforschung, obgleich Fragen von Konflikt, Frieden und gesellschaftlichem Zusammenhalt in der politischen Bildung und in der Öffentlichkeit deutlich an Relevanz zugenommen haben. Umso wichtiger ist es daher, geeignete thematische Schnittstellen zu anderen Forschungsfeldern zu identifizieren und auf diese Weise die interdisziplinäre Kooperation zu stärken. Dies setzt auch ein wechselseitiges Verständnis unterschiedlicher quantitativer und qualitativer Methoden und damit die Sicherung einer breiten Methodenkompetenz im Forschungsfeld voraus.

Drittens bleibt Internationalisierung eine Daueraufgabe, die nicht auf internationale (zumeist englischsprachige) Peer-Review-Publikationen oder auf EU-geförderte Verbundvorhaben reduziert werden sollte. Im Gegenteil: Gerade die Friedens- und Konfliktforschung muss in stärkerem Maße mit Wissenschaftler*innen aus anderen Teilen der Welt interagieren und kooperieren. Dafür bedarf es entsprechender Fellowship-Programme, die (insbesondere jungen) Gastwissenschaftler*innen aus Konfliktregionen einen Aufenthalt an deutschen Einrichtungen ermöglichen - und umgekehrt. Daneben gibt es eine weitere Dimension, die bis dato nur wenig thematisiert worden ist (auch nicht im Bericht des Wissenschaftsrates): Eine stärkere Rolle der Friedens- und Konfliktforschung im Kontext der Außenkultur- und Außenwissenschaftspolitik, um bspw. den Aufbau von Studiengängen und Forschungsinstituten in anderen Ländern zu unterstützen und lokale Kapazitäten zu fördern, was wiederum die internationale Forschungskooperation und Netzbildung verbessert. Dies wird sicherlich auch in Zukunft keine originäre Aufgabe für die DSF sein können, gleichwohl wäre es hier sinnvoll, den Dialog mit entsprechenden Fördereinrichtungen und Akteuren der Außenkulturpolitik (u. a. Auswärtiges Amt, Deutscher Akademischer Austauschdienst) zu suchen.

\section{Fazit und Ausblick}

Der Wissenschaftsrat hat der Friedens- und Konfliktforschung zweifellos ein überaus positives Zeugnis ausgestellt, auch wenn einige Schwächen und Defizite zu 
Recht erwähnt werden. Er würdigt damit die erheblichen Anstrengungen der letzten beiden Jahrzehnte, das Forschungsfeld durch die Stärkung von universitären und auBeruniversitären Einrichtungen sowie durch neue Professuren und Studiengänge zu professionalisieren und strukturell wie thematisch weiterzuentwickeln. Daran hatte auch die DSF einen wesentlichen Anteil.

Das Ergebnis der Evaluation lässt sich zugespitzt wie folgt interpretieren: Die Friedens- und Konfliktforschung ist nunmehr nach einer erfolgreichen Aufbau- und Konsolidierungsphase an einem Punkt angelangt, an dem sie ihre Potenziale aus eigener Kraft - mit den vorhandenen Ressourcen - nicht mehr ausschöpfen kann. Von einer strategischen Entwicklungsperspektive für das Forschungsfeld, die über den Tellerrand der jeweiligen Forschungsinstitute hinausreicht, kann zurzeit kaum die Rede sein. Vielmehr zeigt sich, wie das Beispiel der naturwissenschaftlich-technischen Friedensforschung besonders deutlich vor Augen führt, dass vorhandene, auch international sichtbare Kapazitäten in verschiedenen Themenfeldern wegzubrechen drohen. Auch größere Forschungsverbünde oder ein strategischer Ausbau der Internationalisierung lassen sich nur in Ansätzen realisieren. Es bedarf folglich nachhaltiger Investitionen in die Infrastruktur, Vernetzung und Forschungsförderung, um die Friedens- und Konfliktforschung in ihrer Breite für neue Aufgaben zu wappnen. Der Wissenschaftsrat hat dies erkannt und der Forschungspolitik eine Reihe von Aufgaben ins Heft geschrieben.

Die „Steilvorlage“ des Wissenschaftsrates ist durch den Deutschen Bundestag und die Bundesregierung in ersten konkreten Schritten aufgenommen worden. In der eingangs erwähnten Bundestagsdebatte vom 13.02.2020 kündigte das BMBF an, eine Ausschreibung von Fördermitteln mit einem Gesamtvolumen von 30Mio. $€$ vorzubereiten, mit dem die überregionalen, internationalen und interdisziplinären Vernetzungsstrukturen der Friedens- und Konfliktforschung fortentwickelt werden sollen. Außerdem soll die DSF in den kommenden Jahren mit Zustiftungen aus dem Etat des Auswärtigen Amtes und durch Projektmittel aus dem BMBF unterstützt werden, um sie ,zukunftsfähig und nachhaltig“ aufzustellen. Sollten diese Schritte umgesetzt werden, sind aus Sicht der Stiftung drei wichtige Etappenziele erreicht: Erstens würde die DSF in die Lage versetzt, ihre Arbeit ohne weiteren Kapitalverzehr fortzusetzen. Zweitens könnte die thematische Förderlinie auf absehbare Zeit verstetigt werden; zudem sollte es gelingen, die Förderangebote in der offenen Förderung besser auszustatten und damit für Antragsteller*innen attraktiver zu gestalten. Drittens wäre das Fundament für den moderaten Anstieg des Stiftungskapitals gelegt, ohne dass damit die strukturelle Unterausstattung beseitigt wäre. Dieses Ziel, das nicht zuletzt die Unabhängigkeit und dauerhafte Perspektive der DSF absichern soll, bleibt weiterhin auf der Agenda. ${ }^{11}$

Der Bericht des Wissenschaftsrats hat ohne Zweifel den Anliegen der Friedensund Konfliktforschung, vor allem im parlamentarischen Raum, Türen geöffnet. Dies zeigte sich nicht nur bei der Bundestagsdebatte der Fachpolitiker*innen aus dem Forschungsbereich, bei der sich ein fraktionsübergreifender Konsens abzeichnete. Offenheit und Interesse besteht insbesondere bei den Fachpolitiker*innen der Außen-, Entwicklungs- und Sicherheitspolitik, aber auch bei Abgeordneten, die sich

11 Siehe hierzu auch den Beitrag von Gehring/Rubner (https://doi.org/10.1007/s42597-020-00031-3). 
um Themen der inneren Sicherheit und des gesellschaftlichen Zusammenhalts bemühen. Diese Erfahrungen machen wir immer wieder bei den Parlamentarischen Abenden, die die DSF veranstaltet.

Wer die Mechanismen des politischen Betriebs kennt, dem ist jedoch bewusst, dass die Umsetzung finanzrelevanter Empfehlungen alles andere als ein Selbstläufer ist. Es ist weiterhin wichtig, Gespräche mit politischen Entscheidungsträger*innen zu führen, nicht zuletzt auch mit den Fraktionsführungen sowie, last but not least, mit den Haushaltspolitiker*innen, um auf offene Punkte der Wissenschaftsratsevaluation zu strukturellen Fragen hinzuweisen und für Anliegen und Angebote aus dem Forschungsfeld zu werben. Es wäre aus Sicht der Stiftung sehr erfreulich, wenn es auf der Grundlage der Evaluation gelingt, einen Rückfall in frühere Zeiten zu verhindern, als die DSF und das Forschungsfeld zwar in den Koalitionsverträgen freundliche Erwähnung fanden, die Rolle und der Stellenwert der Friedens- und Konfliktforschung aber zwischen den Koalitionären als ungeklärt oder gar als hochgradig umstritten angesehen wurde. Die positive Evaluation sollte politisch dazu genutzt werden, um die Förderung des Forschungsfeldes und den Kapitalaufbau für die DSF zu einem gemeinsamen Projekt der Bundesregierung und der sie tragenden Fraktionen zu machen. Deshalb wird es wichtig, dass aus dem Forschungsfeld und aus der DSF heraus Konzepte und Ideen entwickelt werden, die den Verantwortlichen in Bund und Ländern aufzeigen, wie der Ausbau konkret und nachhaltig gestaltet werden kann.

Funding Open Access funding provided by Projekt DEAL.

Open Access Dieser Artikel wird unter der Creative Commons Namensnennung 4.0 International Lizenz veröffentlicht, welche die Nutzung, Vervielfältigung, Bearbeitung, Verbreitung und Wiedergabe in jeglichem Medium und Format erlaubt, sofern Sie den/die ursprünglichen Autor(en) und die Quelle ordnungsgemäß nennen, einen Link zur Creative Commons Lizenz beifügen und angeben, ob Änderungen vorgenommen wurden.

Die in diesem Artikel enthaltenen Bilder und sonstiges Drittmaterial unterliegen ebenfalls der genannten Creative Commons Lizenz, sofern sich aus der Abbildungslegende nichts anderes ergibt. Sofern das betreffende Material nicht unter der genannten Creative Commons Lizenz steht und die betreffende Handlung nicht nach gesetzlichen Vorschriften erlaubt ist, ist für die oben aufgeführten Weiterverwendungen des Materials die Einwilligung des jeweiligen Rechteinhabers einzuholen.

Weitere Details zur Lizenz entnehmen Sie bitte der Lizenzinformation auf http://creativecommons.org/ licenses/by/4.0/deed.de.

\section{Literatur}

Bündnis 90/Die Grünen. 2019. „Ein Kompass in unruhigen Zeiten - Friedensforschung als Grundlage der Politik stärken “, Drucksache 19/14111. http://dip21.bundestag.de/dip21/btd/19/141/1914111.pdf.

Deutscher Bundestag Drucksache 18/10849 vom 17. Jan. 2017

Deutscher Bundestag. 2020. Plenarprotokoll 19/146, Seite 18350. http://dipbt.bundestag.de/dip21/btp/19/ 19146.pdf\#P.18350. Zugegriffen: 13.4.2020

Wissenschaftsrat. 2019. Empfehlungen zur Weiterentwicklung der Friedens- und Konfliktforschung. Drs. 7827-19. Gießen: Wissenschaftsrat. 